\title{
BeppoSAX Wide Field Cameras observations of six type I X-ray bursters
}

\author{
R. Cornelisse ${ }^{1,2}$, F. Verbunt ${ }^{2}$, J. J. M. in 't Zand ${ }^{2,1}$, E. Kuulkers ${ }^{1,2, \star}$, J. Heise ${ }^{1}$, R. A. Remillard ${ }^{3}$, M. Cocchi ${ }^{4}$, \\ L. Natalucci ${ }^{4}$, A. Bazzano ${ }^{4}$, and P. Ubertini ${ }^{4}$ \\ 1 SRON National Institute for Space Research, Sorbonnelaan 2, 3584 CA Utrecht, The Netherlands \\ 2 Astronomical Institute, Utrecht University, PO Box 80000, 3508 TA Utrecht, The Netherlands \\ 3 Center for Space Research, Massachusetts Institute of Technology, Cambridge, MA 02139-4307, USA \\ ${ }^{4}$ Istituto di Astrofisica Spaziale (CNR), Area Ricerca Roma Tor Vergata, Via del Fosso del Cavaliere, 00133 Roma, Italy
}

Received 7 November 2001 / Accepted 15 May 2002

\begin{abstract}
We have discovered three certain (SAXJ1324.5-6313, 2S 1711-339 and SAXJ1828.5-1037) and two likely (SAXJ1818.7+1424 and SAXJ2224.9+5421) new thermonuclear X-ray burst sources with the BeppoSAX Wide Field Cameras, and observed a second burst ever from a sixth one (2S 0918-549). Four of them (excluding 2S 1711-339 and 2S 0918-549) are newly detected X-ray sources from which we observed single bursts, but no persistent emission. We observe the first 11 bursts ever from 2S 1711-339; persistent flux was detected during the first ten bursts, but not around the last burst. A single burst was recently detected from 2S 0918-549 by Jonker et al. (2001); we observe a second burst showing radius expansion, from which a distance of $4.2 \mathrm{kpc}$ is derived. According to theory, bursts from very low flux levels should last $\gtrsim 100 \mathrm{~s}$. Such is indeed the case for the last burst from 2S 1711-339, the single burst from SAX J1828.5-1037 and the two bursts from 2S 0918-549, but not for the bursts from SAX J1324.5-6313, SAX J1818.7+1424 and SAX J2224.9+5421. The bursts from the latter sources all last $\sim 20 \mathrm{~s}$. We suggest that SAX J1324.5-6313, SAX J1818.7+1424, SAX J1828.5-1037 and SAX J2224.9+5421 are members of the recently proposed class of bursters with distinctively low persistent flux levels, and show that the galactic distribution of this class is compatible with that of the standard low-mass X-ray binaries.
\end{abstract}

Key words. accretion, accretion disks - binaries: close - stars: individual: SAX J1324.5-6313, SAXJ1818.7+1424, SAX J1828.5-1037, SAX J2224.9+5421, 2S 1711-339, 2S 0918-549 - stars: neutron - X-rays: bursts

\section{Introduction}

About $40 \%$ of the low mass X-ray binaries in our Galaxy occasionally show (so-called type I) X-ray bursts, which are thermonuclear flashes due to unstable helium and/or hydrogen burning of matter accreted on a neutron star surface (for a review see e.g. Lewin et al. 1993). A typical burst shows a fast rise $(\simeq 1 \mathrm{~s})$ and exponential decay, softening during the decay (interpreted as cooling of the neutron star photosphere), and a spectrum which can be well described with blackbody radiation. At the moment, about $70 \mathrm{X}$-ray bursters are known, approximately 20 of which have been discovered with BeppoSAX (e.g., in 't Zand 2001).

Most X-ray bursters are detected with persistent X-ray flux observed before and after bursts. But some sources have been detected during bursts only, with upper-limits on the persistent emission. These limits vary widely, as they depend on the sensitivity of the instrument used. For example, the X-ray sources

Send offprint requests to: $\mathrm{R}$. Cornelisse,

e-mail: R.Cornelisse@sron.nl

* Present address: ESA-ESTEC, Science Operations and Data Systems Division, SCI-SDG, Keplerlaan 1, 2201 AZ Noordwijk, The Netherlands. in the globular clusters Terzan 1 and Terzan 5 were detected with the Hakucho satellite during bursts only, but EXOSAT and ROSAT also detected the persistent emission (Makishima et al. 1981; Warwick et al. 1988; Verbunt et al. 1995).

The study of X-ray bursts serves various purposes. First, applying the theory of X-ray bursts to observations provides information about the neutron star (e.g., its radius when the distance is known) and about its companion (e.g., whether the matter transferred from the companion is hydrogen-rich or not). Second, the bursts unambiguously decide which of the lowmass X-ray binaries contain a neutron star as opposed to a black hole, and provide (an upper limit to) the distance of the binary, from the condition that its luminosity should be less than the Eddington luminosity. Third, new low-mass X-ray binaries may be discovered by the detection of bursts, in cases where the persistent flux is too low. The last two points help in forming a more accurate view of the total number and distribution of low mass X-ray binaries in our Galaxy, and of the fraction that contains a neutron star.

In this paper we describe the observation with the BeppoSAX Wide Field Cameras of six type I burst sources. Four of these are new X-ray sources with a persistent flux 
below the detection threshold of the Wide Field Cameras of $\simeq 10^{-10} \mathrm{erg} \mathrm{cm}^{-2} \mathrm{~s}^{-1}$. The fifth source is a known X-ray source, from which we detect bursts for the first time. The sixth source is also a previously known X-ray source, the first burst of which was recently discovered by Jonker et al. (2001); we describe a second burst from this source and use it to determine its distance. In Sect. 2 we describe the observations and data reduction; the results are described in Sect. 3. Because of their diversity, the sources are discussed in separate subsections, each of which is accompanied by a sub-subsection in which comparison with other observations are made. Finally, in Sect. 4 we discuss some implications of our results for the theory of bursts (Sect. 4.1) and for various sub-populations of the lowmass X-ray binaries with neutron stars (Sect. 4.2).

\section{Observations and data analysis}

Our observations were obtained from mid 1996 to the end of 2001 with the Wide Field Cameras (Jager et al. 1997) on board of the BeppoSAX satellite (Boella et al. 1997). The Wide Field Cameras are two identical coded mask aperture cameras with a $40^{\circ} \times 40^{\circ}$ (full width to zero response) field of view and $\sim 5^{\prime}$ angular resolution. The source location accuracy is between 0.7 and $5^{\prime}$, and the passband is 2 to $28 \mathrm{keV}$. Data are collected with a time resolution of $0.5 \mathrm{~ms}$. The sensitivity depends on the offaxis angle, but is on average a few mCrab in a $10^{5} \mathrm{~s}$ exposure.

The large sky coverage together with the good angular and time resolution make the Wide Field Cameras an excellent experiment to detect fast transient X-ray phenomena at unexpected sky positions and simultaneously study the behavior of a large fraction of the low mass X-ray binary population in our Galaxy.

Roughly $90 \%$ of all observations of the Wide Field Cameras are carried out in the so-called "secondary mode", in which the pointing of the satellite is set for the Narrow Field Instruments. The pointing of the Wide Field Cameras is arbitrary, except for solar angle constraints and the constraint that the two Wide Field Cameras point in opposite directions, perpendicular to the direction of the Narrow Field Instruments. The remaining $10 \%$ of the Wide Field Camera observations are "primary mode" observations, in which one of the two cameras is pointed at the Galactic Center (and the other, thus, at the anti-center).

We search for X-ray bursts in the lightcurve of the total detector (i.e., a superposition of all the sources in the field of view). With this method we detect a burst if the fast fluctuations in the overall count-rate are small $(<10 \%)$, and if the burst lasts 10 to $100 \mathrm{~s}$ and reaches a peak count-rate of at least a few times $10^{-8} \mathrm{erg} \mathrm{cm}^{-2} \mathrm{~s}^{-1}$. If a burst-like event is observed, a sky image is reconstructed by cross-correlating the detector image with the coded mask (Jager et al. 1997). During this reconstruction the background is subtracted automatically. By comparing the sky image with a catalogue of X-ray sources the burst event can be attributed to a known, or previously unknown, X-ray burster. In this way we discovered bursts from SAX J1324.5-6313, SAX J1828.5-1037, SAX J1818.7+1424, SAX J2224.9+5421 and 2S 0918-549. The burst positions of the newly discovered sources are then used to search for persistent emission and other, possibly somewhat fainter, X-ray bursts in all Wide Field Cameras observations of these sources.

During primary mode, lightcurves for individual sources are created and are searched for bursts. In these data we discovered bursts from 2S 1711-339.

The SAX positions for new X-ray sources are also used to generate studies with the RXTE All-Sky Monitor (ASM; Levine et al. 1997), which has smaller cameras than the WFC but typically provides many measurements per day for every $\mathrm{X}$-ray source. Retrospective ASM light curves are obtained by re-fitting the coded mask data as a superposition of the mask shadows for all of the sources in a particular camera exposure (90 s), including the new target of interest. This reprocessing effort yields light curves that track the behavior of X-ray sources with substantially greater sensitivity compared to the threshold for generic identifications of new transients at random sky positions.

\section{Results}

\subsection{New sources}

A single burst was detected at four locations where no X-ray source is known from previous observations, including the ROSAT All Sky Survey (Voges et al. 1999). We conclude that we have detected four new sources and designated them with SAX names in Table 1 .

All new sources are detected only during the burst: the Wide Field Cameras show no persistent emission or other burst between August 1996 and December 2001 in any of the four cases. To compute the upper limits listed in Table 1, we employ absorption columns interpolated from HI maps by Dickey \& Lockman (1990), and a power law spectrum with photon index $\Gamma=1.0$. (This value is typical for burst sources at low luminosities; see for example Sect. 3.3 below.) For a photon index of $\Gamma=2.0$ the flux values would be $70 \%$ lower.

The All-sky Monitor onboard RXTE satellite (RXTE/ASM) scanned the positions of the four new sources. During the course of our analysis the lightcurves of these sources became available. These lightcurves rather uniformly cover a five year period with, most of the time, several $90 \mathrm{~s}$ observations per day and actual exposure times of $1.7 \times 10^{6} \mathrm{~s}$ and $1.8 \times 10^{6} \mathrm{~s}$, respectively. None of the four lightcurves show a clear detection during this period at an upper-limit of $\simeq 2 \times 10^{-10} \mathrm{erg} \mathrm{cm}^{-2} \mathrm{~s}^{-1}(2-10 \mathrm{keV})$ in 7 days of observation.

The lightcurves of the bursts in various passbands are shown in Fig. 1. They have a fast-rise $(\sim 1-3 \mathrm{~s})$ and exponential-like decay (for SAX J1828.5-1037 this is evident mainly at the lower energies). The decay is faster at higher energies, indicative of spectral softening; decay times are listed in Table 1 together with the spectral fits for the bursts.

The burst profile of SAX J1828.5-1037, as shown in Fig. 1, looks rather strange even considering the low statistical quality. But it is precedented by profiles from at least one well established burster (4U 1636-536; van Paradijs et al. 1986). Thus, it would be interesting to observe more bursts from SAX J1828.5-1037 with better sensitivity. 
Table 1. Results of the BeppoSAX Wide Field Cameras observations. For each burst source the table gives the time of the burst, the position with error $\delta$, the total exposure time on the source between August 1996 and December $2001 t_{\text {tot }}$, the exposure time $t_{\text {exp }}$ of the pointing in which the burst was detected, and the hydrogen absorption column $N_{\mathrm{H}}$, persistent flux $F_{\text {pers }}$ between 2-28 keV, and the distance $d$ derived from the burst peak flux. For the bursts the table gives the e-folding times $\tau$ for the total flux, and for the hard and soft energies, where we choose bands $2-x$ and $x-28 \mathrm{keV}$ such that both bands have similar countrates. Spectral fits have been made for counts integrated over $t_{\mathrm{fit}}$; we give the black body temperature $k T_{\mathrm{bb}}$, radius $R$ at distance (limit) $d$, the average flux $F$ in two bands, the bolometric peak flux $F_{\text {peak }}$, and the total burst fluence $E_{\mathrm{b}}$; and the temperature $k T_{\text {brems }}$ and photon index $\Gamma$ for bremsstrahlung and power law fits, respectively. All fluxes are corrected for absorption.

\begin{tabular}{|c|c|c|c|c|c|c|}
\hline & $\begin{array}{c}\text { SAX } \\
\text { J1324.5-6313 }\end{array}$ & $\begin{array}{c}\text { SAX } \\
\mathrm{J} 1818.7+1424 \\
\end{array}$ & $\begin{array}{c}\text { SAX } \\
\text { J1828.5-1037 }\end{array}$ & $\begin{array}{c}\text { SAX } \\
\mathrm{J} 2224.9+5421 \\
\end{array}$ & $\begin{array}{c}2 \mathrm{~S} \\
1711-339 \\
\end{array}$ & $\begin{array}{c}2 \mathrm{~S} \\
0918-549 \\
\end{array}$ \\
\hline \multicolumn{7}{|l|}{ Source parameters } \\
\hline Burst time (MJD) & 50672.151 & 50683.770 & 51988.863 & 51488.454 & b1-11, Table 2 & 51335.049 \\
\hline RA (J2000) & $13^{\mathrm{h}} 24^{\mathrm{m}} 27^{\mathrm{s}}$ & $18^{\mathrm{h}} 18^{\mathrm{m}} 44^{\mathrm{s}}$ & $18^{\mathrm{h}} 28^{\mathrm{m}} 33^{\mathrm{s}}$ & $22^{\mathrm{h}} 24^{\mathrm{m}} 52^{\mathrm{s}}$ & $17^{\mathrm{h}} 14^{\mathrm{m}} 17^{\mathrm{s}}$ & $09^{\mathrm{h}} 20^{\mathrm{m}} 37^{\mathrm{s}}$ \\
\hline $\operatorname{Dec}(\mathrm{J} 2000)$ & $-63^{\circ} 13^{\prime} .4$ & $14^{\circ} 24^{\prime} .2$ & $-10^{\circ} 37 ! 8$ & $+54^{\circ} 21^{\prime} .9$ & $-34^{\circ} 3.3$ & $-55^{\circ} 13.9$ \\
\hline$\delta(99 \%$ confidence. $)$ & 1'.8 & 2'.9 & 2'.8 & 3'.2 & 1.5 & 0.7 \\
\hline$l_{\mathrm{II}}, b_{\mathrm{II}}$ & $306.6,-0.6$ & $42.2,+13^{\circ} .7$ & $20.9,0.2$ & $102.6,-2.6$ & $352^{\circ} .1,+2^{\circ} .8$ & $275^{\circ} .9,-3.8$ \\
\hline$t_{\text {tot }}($ day $)$ & 58 & 31 & 25 & 65 & 66 & 62 \\
\hline$t_{\exp }(\mathrm{ks})$ & 18.5 & 16.2 & 12.7 & 40.2 & 314 & 36 \\
\hline$N_{\mathrm{H}}\left(10^{22}\right.$ atoms $\left.\mathrm{cm}^{-2}\right)$ & $1.5^{a}$ & $0.1^{a}$ & $1.9^{a}$ & $0.5^{a}$ & $1.5^{b}$ & $0.24^{c}$ \\
\hline$F_{\text {pers }}\left(10^{-10} \mathrm{erg} \mathrm{cm}^{-2} \mathrm{~s}^{-1}\right)$ & $<0.80(3 \sigma)$ & $<1.7(3 \sigma)$ & $<1.9(3 \sigma)$ & $<0.35(3 \sigma)$ & $6.3 \pm 0.6$ & $3.8 \pm 0.6$ \\
\hline$d(\mathrm{kpc})$ & $<6.2$ & $<9.4$ & $<6.2$ & $<7.1$ & $<7.5$ & 4.2 \\
\hline \multicolumn{7}{|l|}{ Burst parameters } \\
\hline$\tau_{2-28 \mathrm{keV}}(\mathrm{s})$ & $6.0 \pm 0.1$ & $4.5 \pm 0.1$ & $11.2 \pm 0.6$ & $2.6 \pm 0.2$ & $7.1 \pm 0.2$ & $48.5 \pm 0.2$ \\
\hline $\mathrm{x}$ & 8 & 4 & 7 & 6 & 6 & 5 \\
\hline$\tau_{2-x \mathrm{keV}}(\mathrm{s})$ & $9.7 \pm 0.4$ & $5.7 \pm 0.2$ & $21.5 \pm 1.3$ & $3.9 \pm 0.7$ & $7.6 \pm 0.3$ & $80.6 \pm 0.7$ \\
\hline $\begin{array}{l}\tau_{x-28 \mathrm{keV}}(\mathrm{s}) \\
\text { black-body fit }\end{array}$ & $2.6 \pm 0.2$ & $1.17 \pm 0.04$ & $4.7 \pm 0.6$ & $1.8 \pm 0.3$ & $5.9 \pm 0.3$ & $36.5 \pm 0.2$ \\
\hline$t_{\text {fit }}(\mathrm{s})$ & 9.3 & 6.0 & 25.1 & 4.0 & - & 86.4 \\
\hline$k T_{\mathrm{bb}}(\mathrm{kev})$ & $2.5 \pm 0.2$ & $1.1 \pm 0.14$ & $2.3 \pm 0.2$ & $2.5 \pm 0.3$ & $1.6 \pm 0.1$ & $2.26 \pm 0.05$ \\
\hline$R(\mathrm{~km})$ at $d$ & $4.5 \pm 0.5$ & $24 ._{-8}^{+5}$ & $4.7 \pm 0.9$ & $4.7 \pm 0.5$ & $5.5-11.9$ & $6.3 \pm 0.2$ \\
\hline$F_{2-10 \mathrm{keV}}\left(10^{-8} \mathrm{erg} \mathrm{cm}^{-2} \mathrm{~s}^{-1}\right)$ & $1.23 \pm 0.10$ & $1.02 \pm 0.03$ & $1.08 \pm 0.40$ & $1.00 \pm 0.42$ & $0.4 \pm 0.1^{d}$ & $4.05 \pm 0.27$ \\
\hline$F_{2-28 \mathrm{keV}}\left(10^{-8} \mathrm{erg} \mathrm{cm}^{-2} \mathrm{~s}^{-1}\right)$ & $2.17 \pm 0.07$ & $1.07 \pm 0.05$ & $1.65 \pm 0.73$ & $1.66 \pm 0.89$ & $0.5 \pm 0.1^{d}$ & $6.1 \pm 0.5$ \\
\hline$F_{\text {peak }}\left(10^{-8} \mathrm{erg} \mathrm{cm}^{-2} \mathrm{~s}^{-1}\right)$ & $4.3 \pm 0.2$ & $1.9 \pm 0.1$ & $4.3 \pm 1.6$ & $3.3 \pm 1.5$ & $3.0 \pm 1.0^{d}$ & $9.4 \pm 1.7$ \\
\hline$E_{\mathrm{b}}\left(10^{-7} \mathrm{erg} \mathrm{cm}^{-2}\right)$ & $\simeq 2.6$ & $\simeq 0.86$ & $\simeq 4.3$ & $\simeq 0.67$ & - & $\simeq 52$ \\
\hline$\chi_{v}^{2}$ (d.o.f.) & $1.0(26)$ & $0.6(26)$ & $0.7(26)$ & $1.0(26)$ & $0.9(270)$ & $1.6(26)$ \\
\hline \multicolumn{7}{|l|}{ bremsstrahlung fit } \\
\hline$k T_{\text {brems }}(\mathrm{keV})$ & $56_{-34}^{+47}$ & $5.9 \pm 1.7$ & $44 ._{-22}^{+146}$ & $50 ._{-25}^{+149}$ & - & $64 \pm 14$ \\
\hline$\chi_{v}^{2}$ (d.o.f.) & $1.4(26)$ & $0.5(26)$ & $1.1(26)$ & $1.1(26)$ & - & $9.7(26)$ \\
\hline \multicolumn{7}{|l|}{ power law fit } \\
\hline$\Gamma$ & $1.4 \pm 0.1$ & $2.3 \pm 0.21$ & $1.4 \pm 0.2$ & $1.4 \pm 0.2$ & - & $1.31 \pm 0.04$ \\
\hline$\chi_{v}^{2}$ (d.o.f.) & $1.5(26)$ & $0.5(26)$ & $1.1(26)$ & $1.1(26)$ & - & $10(26)$ \\
\hline
\end{tabular}

${ }^{a}$ Interpolated from Dickey \& Lockman (1990); ${ }^{b}$ From NFI, see Sect. 3.2.1; ${ }^{c}$ From Christian \& Swank (1997); ${ }^{d}$ Values for b8.

To obtain a spectrum we integrate over the burst duration; the exact integration times are given in Table 1 . We fit the spectra with a black-body, power law, and bremsstrahlung model. The black-body gives the best description for three of the four bursts and is acceptable for SAX J1818.7+1424, as expected for type I bursts.

If they are indeed type I bursts, their peak flux must be less than or equal to the Eddington limit of $2 \times 10^{38} \mathrm{erg} \mathrm{s}^{-1}$ (for canonical neutron star values), and we can use the observed peak flux to obtain an upper limit to the distance (e.g. Lewin et al. 1993); these upper limits are also listed in Table 1. We note that due to systematic uncertainties the errors on the distance are $\sim 30 \%$ (e.g. Kuulkers et al. 2002).

To prove that the above-discussed bursts are genuine type I $\mathrm{X}$-ray bursts it must be shown that a black body gives the only acceptable description of their spectrum. From Table 1 we see that this is the case only for SAX J1324.5-6313; for the other three bursts bremsstrahlung or power-law spectra are still acceptable. That is why we consider the two alternative explanations for these three bursts, i.e. that they are stellar X-ray flares or X-ray flashes.

Stellar X-ray flares are generally much longer ( hours) and have much lower peak fluxes than the bursts that we have observed (e.g. Greiner et al. 1994; Haisch \& Strong 1991). We therefore consider it unlikely that any of the three bursts is a stellar flare.

$\mathrm{X}$-ray flashes are related to gamma-ray bursts in the sense that they look like the prompt X-ray counterparts to Gamma ray bursts but lack the $\gamma$-ray emission: they have similar time scales, have the same variety of time profiles (including fastrise exponential-decay shapes), and have spectra that are best described by a power law rather than black body radiation 

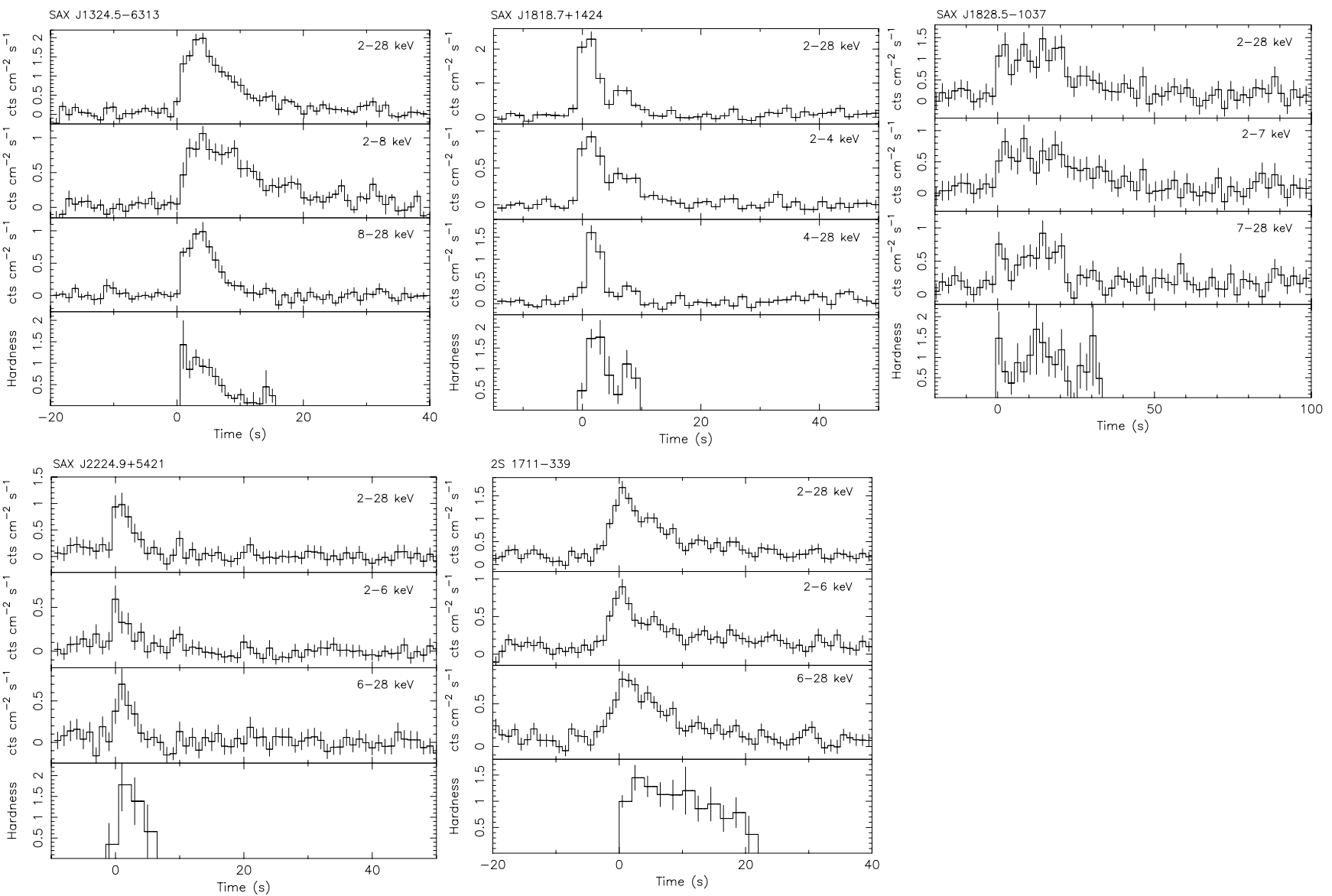

Fig. 1. Lightcurves of bursts from SAX J1324.5-6313, SAX J1818.7+1424, SAX J1828.5-1037, SAX J2224.9+5421, and 2S 1711-339, respectively. For 2S 1711-339 the profile is the average of bursts b1-b10. Three different energy passbands are shown; the total Wide Field Cameras passband $(2-28 \mathrm{keV})$, a low energy passband, and a high energy passband. The high and low energy passbands are chosen in such a way that the count rate was roughly comparable, giving the same statistical quality. Also, the hardness, i.e. the ratio of the high to the low energy count rate, is shown. For each source the binsizes are $1 \mathrm{~s}, 1.5 \mathrm{~s}, 2 \mathrm{~s}, 1 \mathrm{~s}$, and $1 \mathrm{~s}$, respectively; except for the hardness of SAX J2224.9+5421 and 2S 1711-339, which have binsizes of $2 \mathrm{~s}$.

(Heise et al. 2001). X-ray flashes are non-repetitive, and if they are related to gamma-ray bursts no detectable $\mathrm{X}$-ray emission is expected before or lang after the flash. The Wide Field Cameras have observed about 25 of these (in 't Zand et al., in prep.).

SAXJ1828.5-1037 was previously observed during a ROSAT observation (see Sect. 3.1.1), leaving only doubts on the nature of SAX J1818.7+1424 and SAX J2224.9+5421. If these two bursts were X-ray flashes with fast-rise exponentialdecay time profiles, they would be the shortest two of all, and they would be the only ones for which the black body model for the spectrum can not be unambiguously ruled out. Together with the fact that the bursts were at positions near the Galactic plane where type I bursters are likely to occur we think that the most probable explanation for all four bursts is therefore that they are type I X-ray bursts.

\subsubsection{Other observations}

HD 168344, a $V=7.6 \mathrm{mag}$ K2-type star is within the error-radius at a distance of 2 '. from the centroid of SAX J1818.7+1424. There are 18 stars of magnitude 8 or brighter within a $4^{\circ} \times 4^{\circ}$ field around SAXJ1818.7+1424
(ESA 1997). This makes the chance probability of having an 8th magnitude or brighter star within the error radius $0.8 \%$. This probability is so small that we consider the possibility that the event was an X-ray flare from HD 168344.

According to the Tycho Catalogue (ESA 1997) it is a K2 star with $V=7.59$ and $B-V=1.047$, a parallax of $0{ }^{\prime} 0068 \pm 0 . ' 0055$ and a proper motion of about $0.029^{\prime \prime} / \mathrm{yr}$. A main sequence K2 star with this apparent magnitude would have a distance of only $\sim 16 \mathrm{pc}$, incompatible with the small observed parallax. In contrast, a K2 III star of the observed magnitude would be at a distance of 230-270 pc, compatible with the observed parallax, and its velocity perpendicular to the line of sight would be comparable with the observed radial velocity.

The too short burst time scale of $10 \mathrm{~s}$ and the high peak luminosity of $1.2 \times 10^{35} \mathrm{erg} \mathrm{s}^{-1}$ at a distance of $230 \mathrm{pc}$, both exclude that the burst observed by us was a stellar flare on HD 168344. (No stellar flare this short and bright has ever been observed to our knowledge; e.g. Haisch \& Strong 1991.) If a neutron star were a companion of HD 168344, the peak flux of its burst would be $\simeq 6 \times 10^{-4}$ of the Eddington flux. This also is unlikely We conclude that HD 168344 is not the optical counterpart of SAX J1818.7+1424. 


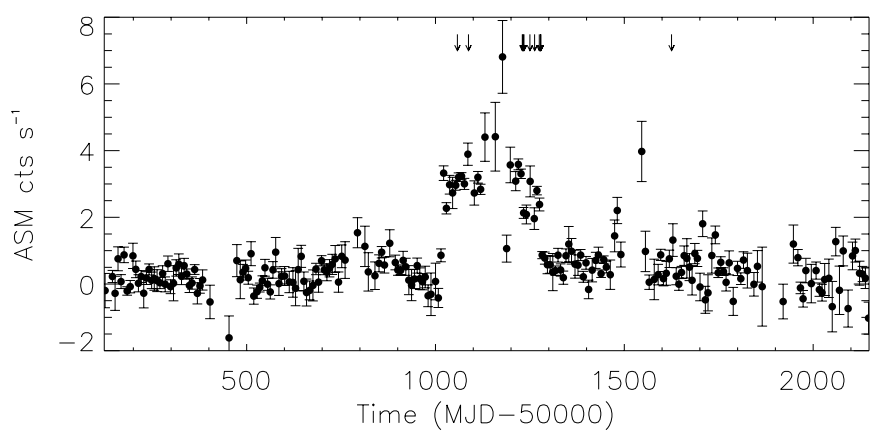

Fig. 2. Long-term lightcurve of 2S 1711-339 obtained with the RXTE All-sky monitor, which shows an outburst between July 1998 and May 1999. Each point represents a one-week average. The vertical arrows indicate the times at which bursts were detected with the BeppoSAX Wide Field Cameras.

Whereas none of the four burst positions coincides with a catalogued ROSAT source, we find that SAX J1828.5-1037 is in the field of view of a $9.4 \mathrm{ks}$ ROSAT/PSPC pointed observation obtained on April 4 1993. We have analyzed this observation and detect seven sources (in channels 50-240), one of which is in the Wide Field Cameras error-circle. With a radius for the ROSAT field of view of $\sim 50^{\prime}$ the chance probability that one of seven sources falls in the SAX J1828.5-1037 errorcircle of 2.8 is about $2 \%$. Thus the ROSAT source is probably the counterpart of SAXJ1828.5-1037, confirming that SAX J1828.5-1037 was not an X-ray flasher. The position of the ROSAT source is RA $=18^{\mathrm{h}} 28^{\mathrm{m}} 25.7^{\mathrm{s}}$, Dec $=-10^{\circ} 37^{\prime} 51^{\prime \prime}$ $(\mathrm{J} 2000)$ with an error of $39^{\prime \prime}(1 \sigma)$. The source is not detected in channels $11-50$, as expected for a highly absorbed source. The countrate of $0.011 \pm 0.002 \mathrm{cts} \mathrm{s}^{-1}$ corresponds to an unabsorbed flux between $0.5-2.5 \mathrm{keV}$ of $1.9 \times 10^{-12} \mathrm{erg} \mathrm{cm}^{-2} \mathrm{~s}^{-1}$ for a power law with photon index $\Gamma=1$. At a distance of $6.2 \mathrm{kpc}$ this corresponds to a luminosity of $8.7 \times 10^{33} \mathrm{erg} \mathrm{s}^{-1}$.

\section{2. $2 S 1711-339$}

2S 1711-339 is an X-ray source that was bright between July 1998 and May 1999. The Wide Field Cameras covered this outburst in August-October 1998 and February-April 1999. Ten short bursts were detected which we designate b1, b2, .., b10. All these bursts appear to have a similar shape. In the left panel of Fig. 3 we show the best example (b5) of these bursts. To fit the average persistent emission before and after the bursts, we employ the absorption column derived in Sect. 3.2.1. A cut-off power law with a photon-index of $0.7 \pm 0.5$ and a high-energy cut off of $2.8 \pm 0.8 \mathrm{keV}$ provides a satisfactory fit; the flux of the persistent emission is given in Table 1.

During a $40 \mathrm{ks}$ observation on March 222000 an eleventh burst (b11) was detected while the persistent emission level before and after the burst was below the detection limit. Assuming that the spectral parameters of the persistent flux observed with bursts b1-b10 still apply, we obtain a $3 \sigma$ upper-limit on the persistent flux of $7.0 \times 10^{-11} \mathrm{erg} \mathrm{cm}^{-2} \mathrm{~s}^{-1}(2-28 \mathrm{keV})$; this is a factor of ten lower than during bursts b1-b10. This burst, which is shown in the right panel of Fig. 3, has a different shape than the previous 10 bursts. Both the rise and decay time for burst
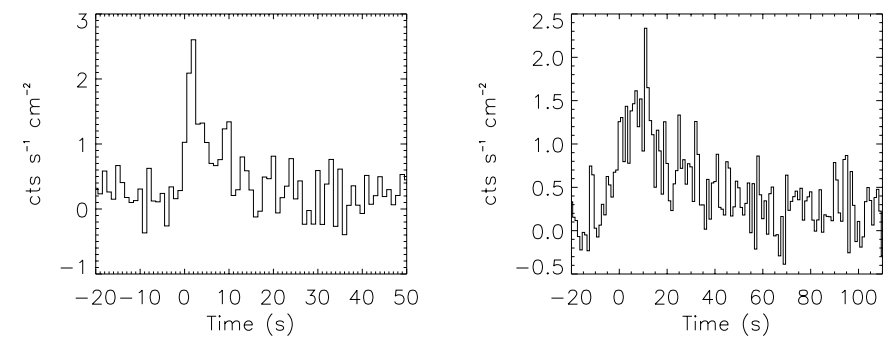

Fig. 3. Bursts b5 (left panel) and b11 (right panel) of 2S 1711-339 in the $2-28 \mathrm{keV}$ passband. The first 10 bursts show similar shapes and are represented by b5. The persistent emission was detected during the observations before/after all these bursts. Before and after b11 the persistent emission was below the detection limit of the Wide Field Cameras.

Table 2. Characteristics of the individual bursts of 2S 1711-339. For each burst we give the time of occurrence, the peak count rate in the WFC, and from a fit of an exponential to the decay, the e-folding decay time and the reduced $\chi^{2}$.

\begin{tabular}{ccccc}
\hline \hline burst & $\begin{array}{c}\text { Start time } \\
(\mathrm{MJD})\end{array}$ & $\begin{array}{c}\mathrm{CR}_{\text {peak }} \\
\left(\mathrm{cm}^{-2} \mathrm{~s}^{-1}\right)\end{array}$ & $\begin{array}{c}\tau_{2-28} \\
(\mathrm{~s})\end{array}$ & $\begin{array}{c}\chi_{v}^{2} \\
47 \text { d.o.f. }\end{array}$ \\
\hline b1 & 51058.024301 & $1.15 \pm 0.05$ & $6.9 \pm 0.4$ & 1.3 \\
b2 & 51087.268967 & $1.73 \pm 0.04$ & $7.0 \pm 0.3$ & 1.5 \\
b3 & 51229.857483 & $1.72 \pm 0.04$ & $6.3 \pm 0.2$ & 1.3 \\
b4 & 51232.083607 & $1.69 \pm 0.04$ & $3.6 \pm 0.1$ & 1.4 \\
b5 & 51234.270915 & $1.63 \pm 0.06$ & $5.2 \pm 0.2$ & 1.6 \\
b6 & 51249.958462 & $0.94 \pm 0.03$ & $14.5 \pm 0.8$ & 1.4 \\
b7 & 51262.473439 & $1.29 \pm 0.03$ & $11.5 \pm 0.4$ & 1.4 \\
b8 & 51274.775982 & $1.88 \pm 0.04$ & $6.9 \pm 0.2$ & 1.5 \\
b9 & 51278.327360 & $1.25 \pm 0.07$ & $10.5 \pm 0.9$ & 1.5 \\
b10 & 51278.992006 & could not be constrained & \\
b11 & 51625.046991 & $1.18 \pm 0.04$ & $15.0 \pm 0.7$ & 1.3 \\
\hline
\end{tabular}

b11 are longer. In Table 2 we summarize the characteristics for the 11 individual bursts. In Fig. 2 we show the RXTE/ASM long-term lightcurve of 2S 1711-339 which shows that the first 10 bursts occurred during an outburst.

It is clear from Table 2 that none of the exponential fits to the decay is formally acceptable. Also, for none of the individual bursts cooling can be unambiguously shown. Therefore, we decided to combine the light curves of burst b1 to b10. The last burst, b11, is excluded because of its deviating shape. To combine the bursts we created lightcurves with a time resolution of $1 \mathrm{~s}$. We took the highest bin as the peak for each burst and the corresponding time as $t=0 \mathrm{~s}$. Then the bursts were combined, by averaging the count rates and determining the statistical error in the mean. Figure 1 shows the resulting profile.

A black-body spectrum was fit to the burst spectrum of all 11 burst. We assumed a fixed absorption column, $N_{\mathrm{H}}=$ $1.5 \times 10^{22}$ atoms cm$~^{-2}$ (see Sect. 3.2.1). We fitted the 11 burst spectra simultaneously; the black-body temperature was forced to be the same for all bursts, whereas the black-body radius was allowed to vary. In Table 1 we have summarized the results for fits to the spectrum and to the exponential decays in different 


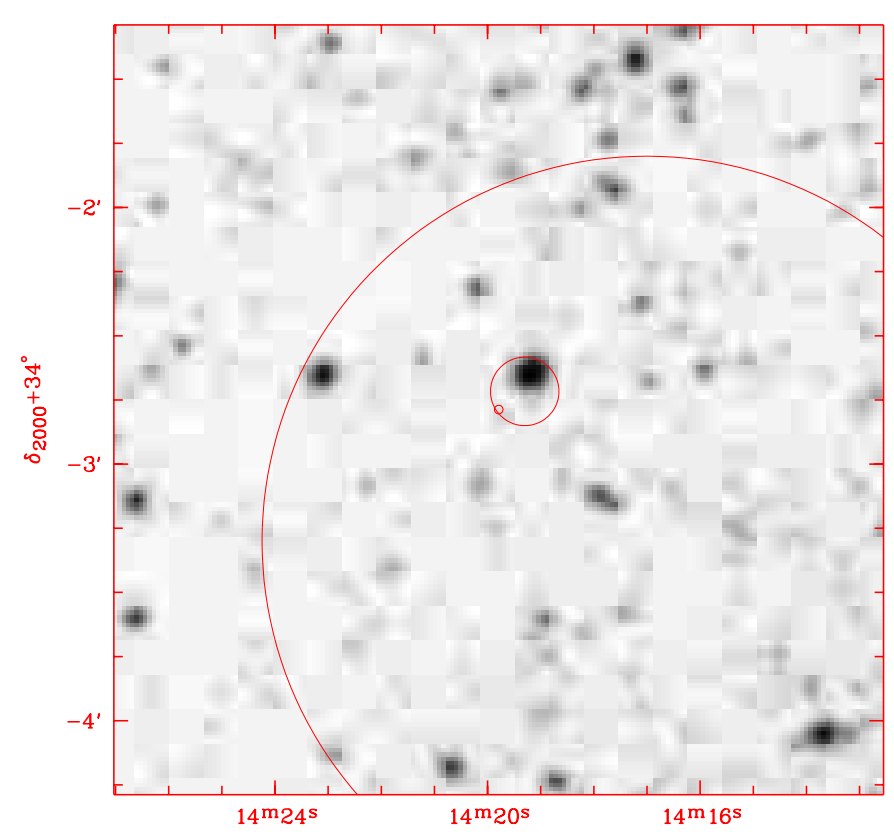

Fig. 4. Error circles (large to small) from the Wide Field Cameras position (this paper), RXS J171419.3-340243 (Voges et al. 1999), and Chandra position of 2S 1711-339 (this paper) superposed on an image from the Digitized Sky Survey.

passbands. Instead of giving the radius of each individual burst we indicate the range of radii in Table 1 . Varying the temperature and forcing the radius to be the same for all bursts simultaneously gives a range of temperatures between $1.2-2.0 \mathrm{keV}$ and a radius of $7.8 \pm 0.9 \mathrm{~km}$ (at $7.5 \mathrm{kpc}$ ). Burst b8 has the highest peak flux. The temperatures and the radii found are typical values for type I bursts. We conclude that the 11 events are type I bursts.

From the observed peak flux during the burst and the constraint that this must be less than the Eddington limit, we derive from the brightest burst b8 an upper limit to the distance of $2 \mathrm{~S} 1711-339$ of $7.5 \mathrm{kpc}$.

\subsubsection{Other observations of $2 \mathrm{~S} 1711-339$}

A Chandra/ACIS-S observation of 2S 1711-339 was performed on June 92000 for a total of 949 s. A single relatively bright source with a countrate of $0.46 \pm 0.02 \mathrm{cts} \mathrm{s}^{-1}$ is detected at a position RA $=17^{\mathrm{h}} 14^{\mathrm{m}} 19.8^{\mathrm{s}}$, Dec $=-34^{\circ} 02^{\prime} 47^{\prime \prime}(\mathrm{J} 2000)$ with a conservative error-radius of $1^{\prime \prime}$.

Our WFC position for 2S 1711-339 (Table 1), the position of the Ariel V source A 1710-34 (Carpenter et al. 1977), and the position of the source RXS J171419.3-340243 from the ROSAT All Sky Survey (Voges et al. 1999) are all compatible with the Chandra position of 2S 1711-339 as illustrated in Fig. 4. We conclude that they are all the same source. We note that the nearest source with comparable (ROSAT) brightness in the ROSAT All Sky Survey is at a distance of $\sim 30^{\prime}$.

The spectrum during a BeppoSAX Narrow Field Instrument (NFI) observation on February 29, 2000 (MJD 51603) of 2S 1711-339 is best described by an absorbed power law model with photon index 2.2 and an absorption column of $1.5 \times 10^{22}$ atoms $\mathrm{cm}^{-2}$ for the persistent emission (Migliari, di Salvo, Belloni, in preparation). Use of this spectrum for the persistent flux measured with the Wide Field Cameras does not significantly change the numbers given in Sect. 3.2 and Table 1. The flux between 2 and $6 \mathrm{keV}$ in units of $10^{-11} \mathrm{erg} \mathrm{cm}^{-2} \mathrm{~s}^{-1}$ for 2S 1711-339 varied from $<40$ in February 1975 to 200 in September 1976 (Carpenter et al. 1977), to 5 during the EXOSAT Galactic Plane Survey (Warwick et al. 1988). Assuming the spectrum as observed by the NFI we obtain fluxes between 2 and $6 \mathrm{keV}$ in units of $10^{-11} \mathrm{erg} \mathrm{cm}^{-2} \mathrm{~s}^{-1}$ of 2 during the ROSAT All Sky Survey, 2.4 during the NFI observations, and 0.3 during the Chandra/ACIS-S observations. The large range of fluxes shows that $2 \mathrm{~S} 1711-339$ is a genuine transient.

\section{3. $2 S 0918-549$}

A single bright burst was detected from the X-ray source 2S 0918-549 on June 6.049, 1999. The burst has a fast rise, a flat top and an exponential decay. In Table 1 we summarize exponential-decay fits in different passbands and the results of spectral modeling. We fix the interstellar column at the value found from Einstein X-ray data (Christian \& Swank 1997).

The persistent flux is satisfactorily described by a cut-off power law spectrum with a photon index of $0.9 \pm 0.6$ and a high-energy cut-off of $5.2 \pm 3.8 \mathrm{keV}$, or a bremsstrahlung spectrum with a temperature of $8.9 \pm 1.8 \mathrm{keV}$ (the flux is only marginally different between the two models). The black-body model gives the best fit to the burst spectrum. We also performed time-resolved spectral fits for the burst. The values of $k T_{\mathrm{bb}}$ and $\mathrm{R}_{\mathrm{bb}}$ are plotted as a function of time in Fig. 5 . We notice that the burst shows the characteristics of a radiusexpansion burst (an increase in the black-body radius, and a drop in the black-body temperature while the flux stays constant). A distance of $4.2 \mathrm{kpc}$ (with an uncertainty of $30 \%$; see e.g. Kuulkers et al. 2002) and a persistent luminosity of $6.8 \times 10^{35} \mathrm{erg} \mathrm{s}^{-1}$ are implied.

\subsubsection{Other observations}

It turns out that $2 \mathrm{~S} 0918-549$ is in the field of view of a 4.8 ks ROSAT PSPC observation of HD 81188, with a countrate (channels $11-240$ ) of $9.83 \pm 0.05 \mathrm{cts} \mathrm{s}^{-1}$. We have analyzed its spectrum and tried to model it with the combination of a $2 \mathrm{keV}$ black body and $2 \mathrm{keV}$ thermal bremsstrahlung spectrum as fitted by Christian \& Swank (1997) to Einstein data of this source. This model is not acceptable. An acceptable fit $\left(\chi_{v}^{2}=1.2,9\right.$ d.o.f. $)$ is obtained for a combination of a $0.1 \mathrm{keV}$ black body and $3.0 \mathrm{keV}$ thermal bremsstrahlung spectrum, absorbed by $N_{\mathrm{H}}=5.0 \times 10^{21}$ atoms $\mathrm{cm}^{-2}$. The ratio of the black-body to the bremsstrahlung flux in the $0.5-2.5 \mathrm{keV}$ band is 1.45 . For this fit the $2-10 \mathrm{keV}$ flux is due to the bremsstrahlung only, and is $2 \times 10^{-10} \mathrm{erg} \mathrm{cm}^{-2} \mathrm{~s}^{-1}$, a factor two below the level observed with the Wide Field Cameras. The flux in the $0.5-20 \mathrm{keV}$ range is $8 \times 10^{-10} \mathrm{erg} \mathrm{cm}^{-2} \mathrm{~s}^{-1}$, a factor four higher than the level observed with Einstein. 


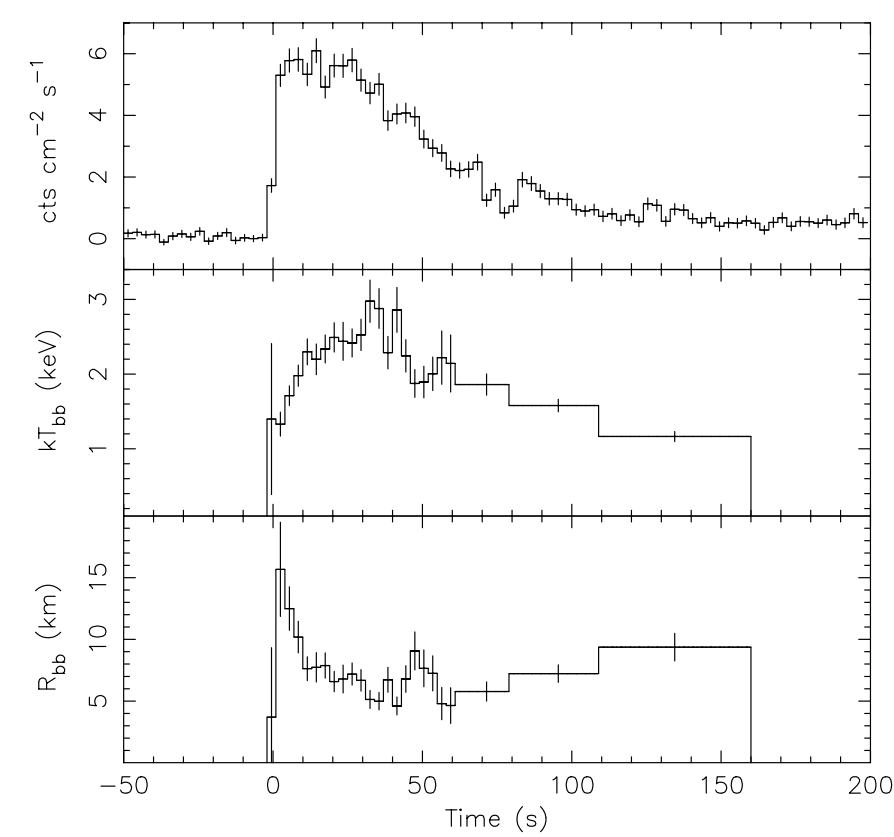

Fig. 5. The lightcurve and results of the time-resolved spectroscopy of the burst from $2 \mathrm{~S} 0918-549$. Each bin is $3 \mathrm{~s}$, apart from the last three bins which are $17 \mathrm{~s}, 30 \mathrm{~s}$ and $50 \mathrm{~s}$ respectively. The top panel gives the lightcurve, the middle panel the black-body temperature during the burst and the bottom panel the black-body radius for a source at $4.2 \mathrm{kpc}$. For the spectral fits a fixed column density of $N_{\mathrm{H}}=0.5 \times$ $10^{22}$ atoms $\mathrm{cm}^{-2}$ is assumed.

Jonker et al. (2001) were the first to detect a burst of $2 \mathrm{~S}$ 0918-549. The data analyzed by Jonker et al. do not combine good time resolution with spectral resolution. Therefore Jonker et al. cannot and indeed do not establish that the burst they observed is at the Eddington-limit. However, they estimate a peak luminosity of about $8.8 \times 10^{-8} \mathrm{erg} \mathrm{cm}^{-2} \mathrm{~s}^{-1}$ (2-20 keV), close to the peak flux of the burst we observed. Accordingly, their distance upper-limit of $4.9 \mathrm{kpc}$ is comparable to our measurement. We conclude that the burst observed by Jonker et al. must be at, or close to, the Eddington limit as well; indeed, the lightcurve presented Jonker et al. is highly suggestive of a radius-expansion burst. The upper-limit $<4.2 \times 10^{-10} \mathrm{erg} \mathrm{cm}^{-2} \mathrm{~s}^{-1}(2-20 \mathrm{keV})$ for the persistent flux before the burst derived by Jonker et al. is compatible with (and close to) the persistent flux that we detect.

The optical counterpart of 2S 0918-549 was identified by Chevalier \& Ilovaisky (1987), as a blue star $(V=21.0$, $B-V=0.3)$ in the error circle of the Einstein position for the $\mathrm{X}$-ray source. The $\mathrm{X}$-ray to optical flux ratio of $\simeq 800$ indicates that the source is a low-mass X-ray binary. Chevalier \& Ilovaisky assume a distance of $15 \mathrm{kpc}$, and note that the X-ray and optical luminosities are low, as compared to those of average low-mass X-ray binaries. Our distance estimate of $4.2 \mathrm{kpc}$ leads to lower luminosities with $M_{\mathrm{V}} \simeq 6.9$ (for $A_{\mathrm{V}} \simeq 1.0$ ), and $L_{\mathrm{x}} \simeq 8.7 \times 10^{35} \mathrm{erg} \mathrm{s}^{-1}$.

The visual extinction is estimated by assuming that $(B-V)_{\mathrm{o}}=0-$ a common value for low-mass X-ray binaries - and is roughly compatible with the value estimated for the $\mathrm{X}$-ray absorption, using the relation between the latter and the visual extinction according to Predehl \& Schmitt (1995).

\section{Discussion}

Our observations add at least three and possibly five bursters to the list of X-ray bursters in our Galaxy and provide a distance estimate for a recently discovered burster. In this section we discuss the implications of our results for the theory of bursts (in Sect. 4.1) and make a comparison between the class of bursters with low persistent luminosities - to which we have added four members - with other low-mass X-ray binaries (in Sect. 4.2).

\subsection{Comparison with burst theory}

We compare the properties of the bursts with theory. Fujimoto et al. (1987, see also Bildsten 2000) propose three classes of bursts. At the lowest accretion rates, $10^{-14} M_{\odot} \mathrm{yr}^{-1} \lesssim \dot{M} \lesssim$ $2 \times 10^{-10} M_{\odot} \mathrm{yr}^{-1}$, a burst is triggered by thermally unstable hydrogen burning, and can last between $10^{2}$ to $10^{4} \mathrm{~s}$. At intermediate accretion rates, $2 \times 10^{-10} \lesssim \dot{M} \lesssim 10^{-9} M_{\odot} \mathrm{yr}^{-1}$, a pure helium burst occurs with a duration of order $10 \mathrm{~s}$. In the high accretion regime, $10^{-9} \lesssim \dot{M} \lesssim 2.6 \times 10^{-8} M_{\odot} \mathrm{yr}^{-1}$, a burst with a duration of tens of seconds may occur in a mixed $\mathrm{He} / \mathrm{H}$ environment. At even higher or lower accretion rates no bursts are expected to occur. To consider our observations, we first converted accretion rates to fractions of the Eddington limit. The pure helium bursts occur when the accretion rate is in the range $0.014-0.070$ of the Eddington accretion rate. The ratio of (the upper limit to) the persistent flux and the peak flux during the burst, where the latter is (a lower limit to) the Eddington flux, provides an estimate of the fraction of the Eddington limit at which a source is accreting. This assumes that the emission is isotropic and that the persistent flux in the range $2-28 \mathrm{keV}$ is close to the bolometric flux. With the values listed in Table 1 we obtain $<0.002$ for SAX J1324.5-6313, <0.009 for SAX J1818.7+1424, <0.004 for SAX J1828.5-1037, <0.001 for SAX J2224.9+5421, 0.02 for 2S 1711-339 at bursts b1-b10, and $<0.002$ at burst b11, and 0.004 for 2S 0918-549.

We thus note that, with the exception of 2S 1711-339 during bursts b1-b10, all sources are in the low accretion regime, and thus according to theory should emit bursts lasting longer than about 100 s. Source 2S 1711-339 follows this prediction nicely, showing short $(\simeq 10-20 \mathrm{~s})$ bursts b1-b10 when accretion was in the intermediate range, and a longer $(\simeq 60 \mathrm{~s})$ burst b11 when the accretion had dropped to the low regime. Also the burst from SAXJ1828.5-1037 lasts about $60 \mathrm{~s}$, pointing towards the low accretion regime. Similarly, the bursts observed by Jonker et al. (2001) and by us for 2S 0918-549 are long $(\simeq 150 \mathrm{~s})$, as predicted from the low accretion rate.

In remarkable contrast, the bursts from SAXJ1324.56313, SAX J1818.7+1424 and SAX J2224.9+5421 are all short ( $\simeq 10-20 \mathrm{~s}$ ), even though these systems appear to be in the low accretion regime. Can it be that the true accretion rate is higher than we estimate? One possibility is that the emission is anisotropic. However, to our knowledge no indication has been found for anisotropies in other burst systems. A second possibility would be that most of the persistent flux is outside the observed 2-28 keV range. However, we estimate that more 
than $50 \%$ of the persistent flux is in this range. We therefore consider it unlikely that these effects are sufficient to bring especially SAX J1324.5-6313 and SAX J2224.9+5421 to the intermediate accretion regime.

A third possibility is that the accretion is limited to a small area of the neutron star, e.g. a ring connected with the accretion disk (Popham \& Sunyaev 2001). This enhances the local accretion rate, which is the parameter determining the properties of the bursts (as discussed by Bildsten 2000). This would imply that the accreting surfaces in SAX J1324.5-6313 and SAX J2224. $9+5421$ are less than about $15 \%$ and $8 \%$ of the surface of the neutron star, respectively. This possibility cannot be excluded a priori, but raises the interesting question why the accreting surface areas would be so different between bursters - the rotation period of the neutron star could affect the area over which the accreted matter spreads out, for example.

A fourth possibility is that the persistent flux at the time of the burst is not representative of the time-averaged flux in the months before the burst. In transients like e.g. Aql X-1, Cen X4, XTE J1709-267 and SAX J1750.8-2900, X-ray bursts were detected during the decline of the outburst, at times when the persistent flux, easily detectable at $L_{\mathrm{x}} \gtrsim 10^{36} \mathrm{erg} \mathrm{s}^{-1}$, was at an accretion rate of ordinary burst sources (Matsuoka et al. 1980; Koyama et al. 1981; Cocchi et al. 1998; Natalucci et al. 1999). Also the transient SAX J1808.4-3658 showed a 100 s long burst 30 days after the peak of an outburst, when the persistent flux had declined below the detection limit of the Wide Field Cameras, $<10^{36} \mathrm{erg} \mathrm{s}^{-1}$ (in 't Zand et al. 2001). However, the RXTE/ASM lightcurves show no detection of SAX J1324.5-6313 and SAX J1818.7+1424, at an upper-limit of $\simeq 10^{36} \mathrm{erg} \mathrm{s}^{-1}$ making a transient outburst very unlikely.

One might propose the possibility that these systems are old and the companion has only pure helium left. In this case only helium bursts can occur independent of the accretion rate. However, calculations on bursts due to pure helium accretion show that at low accretion rates the burst duration increases to $\sim 100 \mathrm{~s}$ (Bildsten 1995).

For pure helium bursts, the energy released during the burst due to nuclear fusion is about $1 \%$ of the accretion energy released when the same matter accreted onto the neutron star before the burst (see e.g. Lewin et al. 1993). From the observed burst fluences and the (upper limits to) the persistent flux, we can therefore derive (lower limits to) the interval to the previous burst. The computed waiting time of $16 \mathrm{~d}$ is sufficiently long to explain that only one burst was detected for $2 \mathrm{~S} 0918-549$, whose WFC exposure times totalled for all observations between August 1996 and December 2001 is about $62 \mathrm{~d}$. For SAX J1324.5-6313, SAX J1818.7+1424, SAX J1828.5-1037 and SAX J2224.9+5421 the total observation times between August 1996 and December 2001 are about 58 d, 30d, 25d and $65 \mathrm{~d}$, respectively. For these sources the waiting times are $>3.7 \mathrm{~d},>0.6 \mathrm{~d},>2.6 \mathrm{~d}$ and $>2.2 \mathrm{~d}$. The chance probability of observing at most one burst for these sources is then $0.07 \%$ or (much) less. The fact that only one burst was observed for each system suggests that the persistent emission levels are much lower than the upper-limits derived.
Table 3. Overview of the burst sources at low persistent emission as observed with the Wide Field Cameras.

\begin{tabular}{|c|c|c|c|c|}
\hline Name & $l_{\mathrm{II}}$ & $b_{\mathrm{II}}$ & $F_{\text {peak }} / F_{\text {pers }}$ & $\tau(\mathrm{s})$ \\
\hline SAX J1324.5-6313 & $306^{\circ} .64$ & -0.59 & $>540$ & 6.0 \\
\hline RX J171824.2-402934 $4^{a}$ & $347^{\circ} .28$ & -1.65 & $>90$ & 47.5 \\
\hline \multirow[t]{3}{*}{ GRS $1741.9-2853^{b}$} & 359.96 & 0.12 & $>130$ & 8.8 \\
\hline & & & $>180$ & 11.0 \\
\hline & & & $>100$ & 16.0 \\
\hline SAX J1752.4-3138 ${ }^{c}$ & $358^{\circ} .44$ & -2.64 & $>120$ & 21.9 \\
\hline SAXJ1753.5-2349 ${ }^{d}$ & 5.30 & 1.10 & $>180$ & 8.9 \\
\hline \multirow[t]{2}{*}{ SAX J1806.5-2215 ${ }^{d}$} & 8.15 & -0.71 & $>200$ & 4.0 \\
\hline & & & $>210$ & 9.0 \\
\hline SAX J1828.5-1037 & 20.88 & +0.18 & $>226$ & 11.2 \\
\hline SAX J1818.7+1424 & $42^{\circ} .32$ & 13.65 & $>110$ & 4.5 \\
\hline SAX J2224.9+5421 & 102.56 & -2.61 & $>940$ & 2.6 \\
\hline
\end{tabular}

${ }^{a}$ Kaptein et al. (2000); ${ }^{b}$ Cocchi et al. (1999); ${ }^{c}$ Cocchi et al. (2001); ${ }^{d}$ in 't Zand et al. (1998).

\subsection{Low persistent emission bursters}

Gotthelf \& Kulkarni (1997) discovered a burst from a lowluminosity source in the globular cluster M28, with a peak luminosity that is only $0.02 \%$ of the Eddington limit. This low peak flux discriminates it from the bursters discussed by Cocchi et al. (2001) and in this paper, that have fluxes close to the Eddington limit: if their peak fluxes were as low as that of the M28 source, they would be a local population near the Sun, which is clearly incompatible with their galactic length and latitude distributions.

As discussed by Cocchi et al. (2001), a class of bursters with low persistent emission has emerged in recent years. The four sources discussed in Sect. 3.1 also appear to be member of this class, strenghtening its existence. Whereas most bursters emit their bursts at persistent luminosities $\gtrsim 10^{36} \mathrm{erg} \mathrm{s}^{-1}$, most of the members of this new class emit bursts at luminosities below the RXTE/ASM detection-limit of $\simeq 10^{36} \mathrm{erg} \mathrm{s}^{-1}$. How much lower is not clear, and we briefly consider three possibilities. One is that the sources are steady in the range $10^{34-35} \mathrm{erg} \mathrm{s}^{-1}$, as suggested for the bursters 1RXS J171824.2-402934 (Kaptein et al. 2000) and SAXJ1828.5-1037 (this paper), whose persistent emission levels were detected at this level with ROSAT a few years before the burst. The second possibility is that the sources are steady at the level $10^{32-33} \mathrm{erg} \mathrm{s}^{-1}$, the quiescent level of soft $\mathrm{X}$-ray transients with neutron stars; and the third possibility is that they are usually at this low level, but emit their bursts during or soon after faint $\left(\lesssim 10^{36} \mathrm{erg} \mathrm{s}^{-1}\right)$ outbursts, as suggested by the case of $2 \mathrm{~S} 1711-339$. More sensitive X-ray observations are required to discriminate between these various possibilities.

However, a first test can be made on the basis of the spatial distributions. In Fig. 6 we compare the distributions of galactic length and latitude for the bursters with low persistent luminosity - listed in Table 3 - with those of the low-mass X-ray binaries. For the latter we use exponential distributions in galactic longitude and latitude with scale angles of $45^{\circ}$ and 8.3 , respectively, as determined by van Paradijs \& White (1995). Kolmogorov-Smirnov tests indicate that the bursters with low 

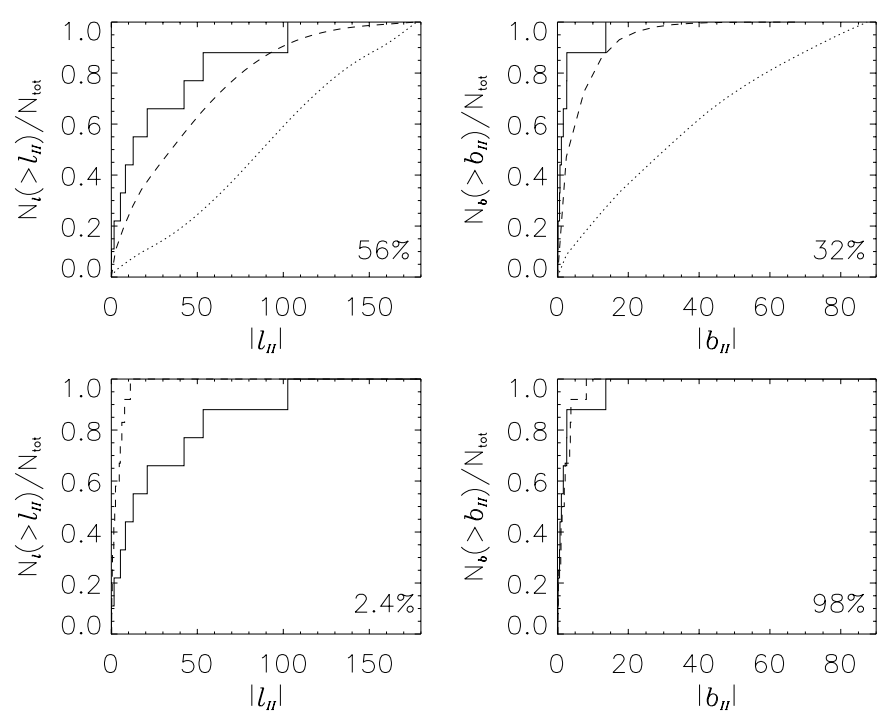

Fig. 6. The top two panels show the cumulative Galactic longitude $l_{\text {II }}$ and latitude $b_{\text {II }}$ distributions (solid lines) of the low persistent emission bursters compared to the exponential (Galactic) distribution of the low-mass X-ray binaries weighted with observation times (dashed lines). The probability according to a two-sided Kolmogorov-Smirnov test that they have the same distribution is given in the lower right corners. For comparison we also show an isotropic distribution weighted with observation times (dotted lines). In the bottom two panels the low persistent emission bursters (solid line) are compared to the faint transients (dashed line).

persistent luminosity may indeed be drawn from the distribution of low-mass X-ray binaries.

A new class of faint transients has been discovered with BeppoSAX: transients whose outbursts are rather fainter (peaking below $10^{37} \mathrm{erg} \mathrm{s}^{-1}$ ) and often also shorter (lasting less than a month) than the outbursts of the ordinary soft X-ray transients which reach the Eddington limit and may last months (Heise et al. 2000). It is tempting to assume that the bursters at low persistent emission are an extension of this class of faint transients. Remarkably, this new class of faint transients is more concentrated towards the galactic center than the ordinary low-mass X-ray binaries (In 't Zand 2001). A Kolmogorov-Smirnov test shows that the galactic longitudes of bursters with low persistent emission cannot be drawn from the longitude distribution of the faint transients, as illustrated in Fig. 6.

We conclude that the bursters at low persistent emission are probably not from the same class as the faint transients, which makes the transient explanation even more unlikely.

Acknowledgements. We thank Gerrit Wiersma for discussions on the Wide Field Cameras exposures. We thank Darragh O'Donoghue for optical observations of the bright star near the position of 2S 1711-339. The BeppoSAX satellite is a joint Italian and Dutch program. We made use of quick-look results provided by the ASM/RXTE team.

\section{References}

Bildsten, L. 1995, ApJ, 438, 852

Bildsten, L. 2000, in Cosmic explosions, AIP, ed. S. Holt, \& W. Zhang, E65

Boella, G., Butler, R., Perola, G., et al. 1997, A\&AS, 122, 299

Carpenter, G. F., Eyles, C. J., Skinner, G. K., Wilson, A. M., \& Willmore, A. P. 1977, MNRAS, 179, 27

Chevalier, C., \& Ilovaisky, S. A. 1987, A\&A, 172, 167

Cocchi, M., Bazzano, A., Natalucci, L., et al. 1998, ApJ, 508, L163

Cocchi, M., Bazzano, A., Natalucci, L., et al. 1999, A\&A, 346, L45

Cocchi, M., Bazzano, A., Natalucci, L., et al. 2001, A\&A, 378, L71

Christian, D. J., \& Swank, J. H. 1997, ApJS, 109, 177

Dickey, J. M., \& Lockman, F. J. 1990, ARA\&A, 28, 215

ESA 1997, The Hipparcos and Tycho Catalogues, ESA SP-1200

Fujimoto, M. Y., Sztajno, M., Lewin, W. H. G., \& van Paradijs, J. 1987, ApJ, 319, 902

Gotthelf, E., \& Kulkarni, S. 1997, ApJ, 490, L161

Greiner, J., Duerbeck, H. W., \& Gershberg, R. E. (eds.) 1994, Flares and Flashes (Berlin: Springer)

Haisch, B., \& Strong, K. T. 1991, ARA\&A, 29, 275

Heise, J., in 't Zand, J. J. M., \& Kuulkers, E. 2000, HEAD 32, 28.03

Heise, J., In 't Zand, J. J. M., Kippen, R. M., \& Woods, P. M. 2001, in Gamma-Ray Bursts in the Afterglow Era, ed. E. Costa, F. Frontera, \& J. Hjorth, ESO Astrophys. Symp. Ser., 16

in 't Zand, J. J. M., Heise, J., Muller, J. M., et al. 1998, Nucl. Phys. B, 69, 228

in 't Zand, J. J. M. 2001, in Exploring the gamma-ray universe, ed. A. Gimenez, V. Reglero, \& C. Winkler, ESA Pub. Div., 463

in 't Zand, J. J. M., Cornelisse, R., Kuulkers, K., et al. 2001, A\&A, 372,916

Jager, R., Mels, W., Brinkman, A., et al. 1997, A\&AS, 125, 557

Jonker, P. G., van der Klis, M., Homan, J., et al. 2001, ApJ, 553, 335

Kaptein, R. G., in 't Zand, J. J. M., Kuulkers, E., et al. 2000, A\&A, 358, L71

Koyama, K., Inoue, H., Makishima, K., et al. 1981, ApJ, 247, L27

Kuulkers, E., Homan, J., van der Klis, M., et al. 2002, A\&A, 382, 947

Levine, A. M., Bradt, H., Cui, W., et al. 1996, ApJ, 469, L33

Lewin, W., van Paradijs, J., \& Taam, R. 1993, Space Sci. Rev., 62, 223

Makishima, K., Ohashi, T., Inoue, H., et al. 1981, ApJ, 247, L23

Matsuoka, M., Inoue, H., Koyama, K., et al. 1980, ApJ, 240, L137

Natalucci, L., Cornelisse, R., Bazzano, A., et al. 1999, ApJ, 523, L45

van Paradijs, J., Sztajno, M., Lewin, W. H. G., et al. 1986, MNRAS, 221,617

van Paradijs, J., \& White, N. 1995, ApJ, 447, 33

Popham, R., \& Sunyaev, R. 2001, ApJ, 547, 355

Predehl, P., \& Schmitt, J. H. M. M. 1995, A\&A, 293, 889

Verbunt, F., Bunk, W., Hasinger, G., \& Johnston, H. M. 1995, A\&A, 300,732

Voges, W., Aschenbach, B., Boller, T., et al. 1999, A\&A, 349, 389

Warwick, R. S., Norton, A. J., Turner, M. J. L., Watson, M. G., \& Willingale, R. 1988, MNRAS, 232, 551 